\section{Reprodutibilidade interavaliadores da identificação da redução da cadência em exercícios de força}

\section{Inter-rater reliability of reduction in cadence identification during resistance exercises}

\author{
Jacilene Guedes de Oliveira ${ }^{1,2}$ \\ Anderson José Melo Rodrigues-Silva 2,3 \\ Thaliane Mayara Pessôa dos Prazeres ${ }^{2,3}$ \\ Marcos André Moura dos Santos \\ Crivaldo Gomes Cardoso Júnior ${ }^{4}$ \\ Raphael Mendes Ritti-Dias ${ }^{2,3}$
}

\section{Resumo}

O objetivo deste estudo foi verificar a concordância interavaliadores na identificação da redução da cadência em exercícios de força. Dez universitários, com experiência prévia em treinamento de força realizaram 3 séries até a fadiga concêntrica, com 2 minutos de intervalo em 6 exercícios (leg press $45^{\circ}$, supino horizontal, extensão do joelho, remada baixa, flexão do joelho e elevação frontal). Dois avaliadores independentes registraram o momento da redução da cadência. Para a análise estatística utilizou o teste de Wilcoxon e o Coeficiente de correlação intraclasse (CCI), $\operatorname{com} p<0,05$. Verificou-se que a redução da cadência identificada pelos dois avaliadores foi similar em todos os exercícios ( $p>0,05$ ). O CCI variou de 0,77 a 0,96. Conclui-se que a identificação da redução da cadência nos exercícios de força apresenta boa reprodutibilidade interavaliadores.

\section{Palavras-chave}

Treinamento de resistência; Fadiga Muscular; Exercício.

\begin{abstract}
The aim of this study was to analyze the inter-rater reliability of the reduction of cadence identification. Ten subjects with previous experience in strength training performed three sets in six exercises (leg press $45^{\circ}$, bench press, leg extension, low rowing, seated leg curl and frontal raises) until concentric failure. Two independent raters analyzed the moment of the reduction in cadence. Statistical analysis was performed with Wilcoxon test and the intraclass correlation coefficient (ICC), with $p<0.05$. The identification of the reduction in cadence was similar between raters in all exercises. The ICC ranged from 0,77 to 0,96. In conclusion, the results of this study suggests that the identification of the reduction in cadence present good inter-rater reliability.
\end{abstract}

\section{Keywords}

Resistance training; Muscle fatigue; Exercise.
Rev Bras Ativ Fis Saúde p. 512-518 DOI: http://dx.doi.org/10.12820/23171634.2012v17n6p512

1 Escola Superior de Educação Física, Universidade de Pernambuco, Recife, PE, Brasil.

2 Grupo de Pesquisa em Hemodinâmica e Metabolismo do exercício, Escola Superior de Educação Física, Universidade de Pernambuco, Recife, PE, Brasil.

3 Programa Associado de Pós-Graduação em Educação Física - PAPGEF. Universidade de Pernambuco/ Universidade Federal da Paraíba, Recife, PE, Brasil.

4 Centro de Educação Física e Desportos, Universidade Estadual de Londrina, Londrina, $P R$, Brasil 


\section{INTRODUÇÃO}

Nos últimos anos o exercício de força vem se popularizando devido aos seus benefícios para saúde de indivíduos com e sem doenças crônicas não transmissíveis ${ }^{1}$. Mais especificamente com relação às doenças cardiovasculares ${ }^{2}$, essa modalidade de exercício tem sido recomendada por promover melhoria na aptidão músculo esquelética ${ }^{3}$, sendo indicada para indivíduos com diferentes doenças tais como a hipertensão arterial, a insuficiência cardíaca e a doença arterial periférica ${ }^{1}$.

Em indivíduos hipertensos, a realização do exercício de força pode aumentar o risco cardiovascular, visto que durante a sua execução a pressão arterial (PA) aumenta acentuadamente ${ }^{4}$. De fato, em estudo clássico realizado com fisiculturistas, foram reportados picos de PA sistólica e diastólica da ordem de 480 e 350 $\mathrm{mmHg}{ }^{5}$, respectivamente, durante a realização do exercício leg press até à fadiga concêntrica. Devido a isso, tem sido recomendado que a prescrição do exercício de força para o hipertenso seja elaborada de forma a minimizar os aumentos de PA durante a realização do exercício.

Nesse sentido, para atenuar os aumentos de PA, a VI Diretrizes Brasileiras de Hipertensão Arterial ${ }^{6}$ recomenda que a realização do exercício de força seja interrompida no momento da redução da cadência. De fato, resultados de um estudo indicaram que a interrupção do exercício de força na redução da cadência, observada visualmente por um avaliador, pode minimizar em $25 \mathrm{mmHg}$ os aumentos da PA durante o exercício ${ }^{7}$. Consequentemente, essa estratégia parece ser efetiva para reduzir o risco cardiovascular durante o exercício de força ${ }^{8}$.

Todavia, a identificação da redução da cadência tem sido feita de acordo com a análise visual de um observador e os dados referentes à qualidade metodológica dessa técnica ainda não foram estabelecidos. Nesse sentido, a análise da reprodutibilidade interavaliadores é um procedimento importante, visto que pode indicar à clareza com que avaliadores distintos identificam à redução da cadência, o que fundamentaria, pelo menos em parte, a realização dessa técnica. Assim, o objetivo do presente estudo foi verificar a reprodutibilidade interavaliadores na identificação da redução da cadência em exercícios de força.

\section{MÉTODOS}

\section{Desenho do estudo}

Trata-se de estudo descritivo com delineamento transversal

\section{Sujeitos}

A amostra foi composta por 10 estudantes ( 8 homens e 2 mulheres) universitários fisicamente ativos, com faixa etária entre 18 e 30 anos, com experiência prévia em exercícios de força, recrutados na Universidade. Foram incluídos no estudo apenas sujeitos eutróficos. Todos foram convidados pessoalmente a participar voluntariamente do estudo, sem nenhum vínculo obrigatório e foram informados quanto aos procedimentos que seriam adotados no estudo. Todos os voluntários assinaram o Termo de Consentimento Livre e Esclarecido. Esse estudo foi aprovado pelo Comitê de Ética da Universidade (protocolo 277/10).

\section{Medidas}

- Avaliação antropométrica

Foram realizadas medidas da massa corporal e estatura. A massa corporal foi ob- 
tida através de balança de plataforma (Welmy, Brasil), com carga máxima de 150 $\mathrm{kg}$ e escala de precisão de $0,1 \mathrm{~kg}$, com os sujeitos descalços e usando roupas leves posicionados no centro da balança. Para a medida da estatura foi usado um estadiômetro de madeira com precisão de $0,1 \mathrm{~cm}$. O índice de massa corporal (IMC) foi calculado pelo quociente entre massa corporal $(\mathrm{kg})$ e o quadrado da estatura $(\mathrm{m})$.

\section{- Sessão experimental}

A sessão experimental foi composta por seis exercícios (leg press $45^{\circ}$, supino horizontal, extensão do joelho, remada baixa, flexão do joelho e elevação frontal), realizados em três séries, até a fadiga concêntrica. A carga em cada exercício foi prescrita com base na massa corporal dos indivíduos. O percentual da massa corporal utilizado em cada exercício foi determinado em estudo piloto realizado previamente as coletas. Assim, os seguintes percentuais da massa corporal foram utilizados: leg press $45^{\circ}$ (217\%), supino horizontal (86\%), extensão do joelho (84\%), remada baixa (105\%), flexão do joelho (113\%), elevação frontal (20\%).

Cada exercício foi iniciado com aquecimento de 10 repetições com 50\% da carga a ser utilizada. Em seguida, os indivíduos realizaram três séries nos seis exercícios propostos, com dois minutos de intervalo entre séries e exercícios. Em cada série, os voluntários foram orientados a manter a cadência do movimento. A série só era interrompida quando o indivíduo chegava à exaustão.

Dois avaliadores independentes registraram o número máximo de repetições e o número de repetições no momento da redução cadência (fadiga moderada) em cada série. Os registros foram feitos individualmente, e em seguida, enviados para uma terceira pessoa que fez a tabulação dos dados.

\section{Análise Estatística}

Previamente ao início da análise, a normalidade e a homogeneidade de variância dos dados foram analisados através dos testes de Shapiro-wilk e de Levene respectivamente. Para verificar diferenças entre os avaliadores, bem como o grau de concordância no momento da redução da cadência, foi aplicado o teste de Wilcoxon e o Coeficiente de correlação intraclasse (CCI), respectivamente. Foi realizada análise de Bland-Altman para determinação das diferenças médias e dos limites de concordância. Em todas as análises foi considerado significante o valor de $\mathrm{p}<0,05$. Os dados não paramétricos são apresentados em mediana e amplitude interquartil, enquanto os dados paramétricos em média e desvio padrão.

\section{RESULTADOS}

Todos os voluntários que participaram do estudo eram jovens e eutróficos. As características gerais dos participantes do estudo estão apresentadas na tabela 1.

$\mathrm{Na}$ tabela 2 são apresentados os valores absolutos do peso levantado nos seis exercícios.

$\mathrm{Na}$ análise interavaliadores, observou-se coeficiente de correlação intraclasse interavaliadores variando de 0,77 a 0,96 (leg press $45^{\circ} \mathrm{r}=0,96$; supino horizontal $r=0,94$; extensão do joelho $r=0,95$; remada baixa $r=0,85$; flexão do joelho $r=$ 0,77 e elevação frontal $r=0,90$ ).

$\mathrm{Na}$ maioria dos exercícios o número de repetições variou entre 7 e 8 , exceto para o exercício de supino horizontal que apresentou uma média de 5 repetições. Os valores médios do número de repetições no momento da redução da cadência obtidos pelo avaliador 1 e pelo avaliador 2 não apresentaram diferença signifi- 
Tabela 1 - Características dos sujeitos

\begin{tabular}{lc}
\hline Variáveis & Média \pm Desvio Padrão \\
\hline Idade (anos) & $22,6 \pm 2,7$ \\
\hline Massa corporal $(\mathrm{kg})$ & $66,8 \pm 9,1$ \\
\hline Estatura $(\mathrm{m})$ & $1,7 \pm 0,1$ \\
\hline Índice de massa corporal $\left(\mathrm{kg} / \mathrm{m}^{2}\right)$ & $22,4 \pm 2,5$ \\
\hline
\end{tabular}

Tabela 2 - Peso total em cada exercício executado

\begin{tabular}{lc}
\hline Exercícios & Média \pm Desvio Padrão \\
\hline Leg press $45^{\circ}(\mathrm{kg})$ & $114,0 \pm 38,3$ \\
\hline Supino horizontal $(\mathrm{kg})$ & $42,0 \pm 19,2$ \\
\hline Extensão do joelho $(\mathrm{kg})$ & $27,6 \pm 13,4$ \\
\hline Remada baixa $(\mathrm{kg})$ & $50,7 \pm 19,9$ \\
\hline Flexão do joelho $(\mathrm{kg})$ & $26,7 \pm 13,0$ \\
\hline Elevação frontal $(\mathrm{kg})$ & $12,4 \pm 4,3$ \\
\hline
\end{tabular}
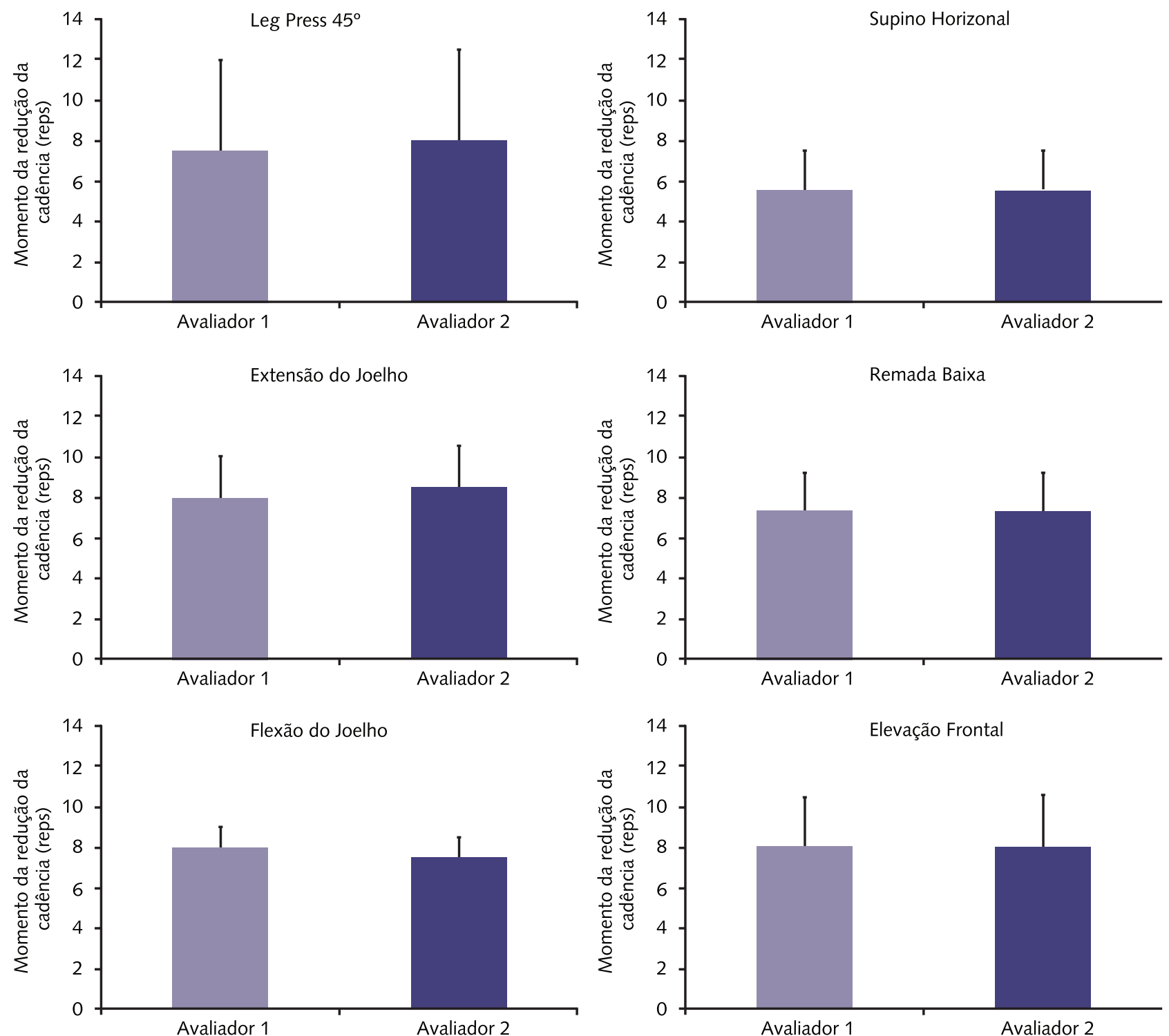

Figura 1 - Concordância interavaliadores do número de repetições no momento da redução da cadência no exercício de força obtidos pelo avaliador 1 e avaliador 2 . 
cantes em nenhum dos exercícios analisados (figura 1). Além disso, o tamanho do efeito em todos os exercícios foi inferior a 0,19 , indicando que a diferença entre os observadores foi pequena.

$\mathrm{Na}$ figura 2 são apresentadas as plotagens de Bland-Altman em cada exercício. Em todos os exercícios a diferença média das diferenças entre os avaliadores foi inferior a uma repetição.
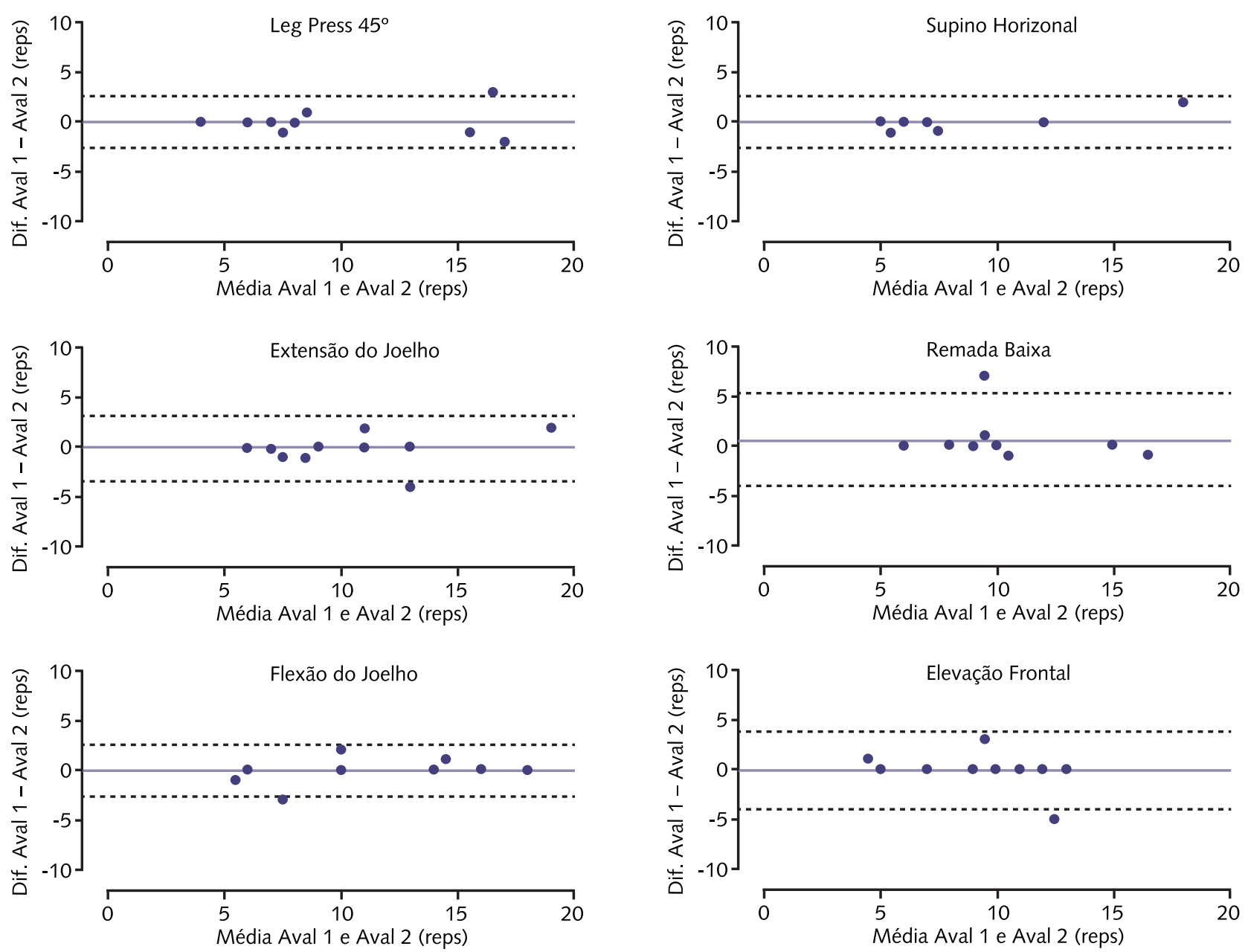

Figura 2 - Número de repetições no momento da redução da cadência obtidos pelo avaliador 1 e avaliador 2 .

\section{DISCUSSÃO}

Os resultados do presente estudo indicaram que a análise visual da redução da cadência durante os exercícios de força por dois avaliadores independentes promoveu resultados similares entre os avaliadores. De fato, o número de repetições identificadas até o momento da redução da cadência foi similar entre os avaliadores. Além disso, a concordância entre os avaliadores ocorreu em todos os exercícios analisados, que contemplavam exercícios para membros superiores, tronco e membros inferiores. Esses resultados sugerem que mesmo se tratando de um indicador subjetivo, o momento em que ocorre a redução da cadência parece ser claro o suficiente para que seja identificado com precisão nos diferentes exercícios.

Apesar de todos os exercícios analisados apresentarem coeficientes de reprodutibilidade satisfatórios, os exercícios de remada baixa e flexão do joelho mostraram correlação inferior aos demais exercícios com CCI de 0,85 e 0,77 respectivamente. 
Possivelmente, a diferença desses resultados pode estar relacionada à própria característica do exercício, bem como limitações nas instalações dos equipamentos utilizados. No exercício de remada baixa há uma oscilação do tronco do indivíduo devido à ausência de encosto para a estabilização, o que pode dificultar a percepção visual da redução da cadência. Em relação ao exercício de flexão do joelho, a máquina apresentava limitação na sua amplitude de movimento quando a carga utilizada era elevada, pois o voluntário não conseguia manter o tronco apoiado no encosto. Além disso, o apoio do tornozelo não disponibilizava estabilidade total para uma perfeita execução do movimento. Esses resultados sugerem que os equipamentos utilizados, especialmente com relação à estabilização do tronco e das articulações, podem prejudicar a análise da redução da cadência.

As análises de Bland-Altman revelaram que para todos os exercícios a média das diferenças entre os avaliadores foi inferior a uma repetição. Além disso, observa-se que a grande maioria dos pontos ficou próxima de zero, o que indica boa concordância entre os avaliadores. Todavia, é importante destacar que um outlier foi observado em todos os exercícios, e na maioria dos exercícios isso ocorreu nas faixas mais altas de repetições. Dessa forma, parece que a identificação da redução da cadência é mais clara em faixas de repetições inferiores a 15. Em termos práticos esse resultado sugere que se o objetivo é prescrever o exercício de força até o momento da redução da cadência é interessante que a prescrição seja feita com menos de 15 repetições.

No presente estudo não foi utilizado metrônomo para padronizar a cadência dos movimentos. Embora a utilização desse instrumento pudesse fornecer um indicador direto da cadência do movimento, isso limitaria a aplicabilidade prática dos resultados, visto que esse procedimento não é realizado na rotina das academias. Além disso, sabe-se que cada indivíduo possui uma cadência natural que tende a ser mantida ao longo do exercício, e a imposição de uma cadência padronizada a todos os indivíduos poderia dificultar a realização dos exercícios. Devido a esses aspectos, nesse estudo optou-se por recomendar aos indivíduos a manutenção de sua cadência natural, o que aumentou a validade externa dos procedimentos empregados.

Esse estudo apresenta algumas limitações que devem ser consideradas. A interrupção do exercício de força na redução da cadência tem sido recomendada para indivíduos hipertensos, enquanto que o presente estudo foi realizado com jovens normotensos. Dessa forma, não é possível extrapolar os resultados deste estudo para hipertensos, de forma que pesquisas futuras devem ser realizadas nessa população. Os exercícios foram prescritos com uma carga moderada, e o quanto a identificação visual da redução da cadência é reprodutível utilizando cargas maiores ou menores não pôde ser estabelecido. Foram incluídos indivíduos de ambos os sexos na amostra, e uma análise estratificada por sexo não pode ser realizada devido ao pequeno número de mulheres no estudo.

\section{CONCLUSÕES}

Os resultados deste estudo indicaram que a identificação visual da redução da cadência durante os exercícios de força foi similar quando analisada por avaliadores independentes.

\section{Agradecimentos}

Agradecemos a todos os voluntários. Esta pesquisa foi financiada pela Coordenação de Aperfeiçoamento de Pessoal de Nível Superior (CAPES) e do Programa de Fortalecimento Acadêmico da Universidade de Pernambuco (PFA-UPE). 


\section{Contribuição dos Autores}

Todos os autores contribuíram de forma significativa para a elaboração do manuscrito, e a versão final lida e aprovada por todos os autores, que afirmam que os dados do manuscrito são verdadeiros.

\section{REFERÊNCIAS}

1. Williams MA, Haskell WL, Ades PA et al.. Resistance exercise in individuals with and without cardiovascular disease: 2007 update: a scientific statement from the American Heart Association Council on Clinical Cardiology and Council on Nutrition, Physical Activity, and Metabolism. Circulation 2007;116:572-584.

2. Pollock ML, Barry AF, Gary JB et al.. Resistance exercise in individuals with and without cardiovascular disease: benefits, rationale, safety, and prescription: An advisory from the Committee on Exercise, Rehabilitation, and Prevention, Council on Clinical Cardiology, American Heart Association; Position paper endorsed by the American College of Sports Medicine. Circulation 2000;101:828-833.

3. Dias RMR, Gurjão ALD, Marucci MDFN. Benefícios do treinamento com pesos para aptidão física de idosos Strength training benefits on the physical fitness of elderly indi-viduals. Acta Fisiátrica 2006;13:90-95.

4. Nery SS, Gomides RS, Giovanio VS et al.. Intra-arterial blood pressure response in hypertensive subjects during low- and high-intensity resistance exercise. Clinics 2010;65: 271-277.

5. MacDougall JD, Tuxen D, Sale DG et al.. Arterial blood pressure response to heavy resistance exercise.J Appl Physiol 1985;58:785-790.

6. Sociedade Brasileira de Cardiologia / Sociedade Brasileira de Hipertensão/Sociedade Brasileira de Nefrologia. VI Diretrizes Brasileiras de Hipertensão. Arq Bras Cardiol 2010; 95:1-51.

7. Gomides RS, Nery SS , Mion Júnior D et al. . Pressão arterial durante o exercício resistido de diferentes intensidades em indivíduos hipertensos. Coleção Pesquisa em Educação Física 2007;6:435-442.

8. Haykowsky MJ, Findlay JM, Ignaszewski AP. Aneurysmal subarachnoid hemorrhage associated with weight training: three case reports. Clin J Sport Med 1996;6:52-55.

9. Duffey MJ, Challis JH. Fatigue effects on bar kinematics during the bench press. J Strength Cond Res 2007;21:556-5

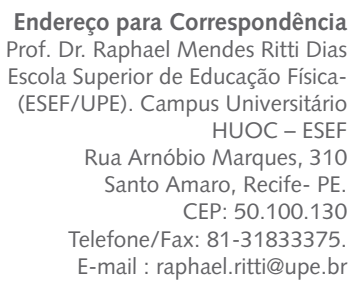

Endereço para Correspondência Prof. Dr. Raphael Mendes Ritti Dias Escola Superior de Educação Física(ESEF/UPE). Campus Universitário HUOC - ESEF Rua Arnóbio Marques, 310 Santo Amaro, Recife- PE. CEP: 50.100 .130 Telefone/Fax: 81-31833375. E-mail : raphael.ritti@upe.br

Recebido 31/01/2013

Revisado 08/02/2013

Aprovado 08/02/2013 